Article

\title{
Autoparallel vs. Geodesic Trajectories in a Model of Torsion Gravity
}

\section{Luis Acedo}

Instituto Universitario de Matemática Multidisciplinar, Universitat Politècnica de València, Building 8G, $2^{\circ}$ Floor, Camino de Vera 46022, Valencia, Spain; E-Mail: luiacrod@imm.upv.es Academic Editor: Lorenzo Iorio

Received: 14 October 2015 / Accepted: 13 November 2015 / Published: 25 November 2015

\begin{abstract}
We consider a parametrized torsion gravity model for Riemann-Cartan geometry around a rotating axisymmetric massive body. In this model, the source of torsion is given by a circulating vector potential following the celestial parallels around the rotating object. Ours is a variant of the Mao, Tegmark, Guth and Cabi (MTGC model) in which the total angular momentum is proposed as a source of torsion. We study the motion of bodies around the rotating object in terms of autoparallel trajectories and determine the leading perturbations of the orbital elements by using standard celestial mechanics techniques. We find that this torsion model implies new gravitational physical consequences in the Solar system and, in particular, secular variations of the semi-major axis of the planetary orbits. Perturbations on the longitude of the ascending node and the perihelion of the planets are already under discussion in the astronomical community, and if confirmed as truly non-zero effects at a statistically significant level, we might be at the dawn of an era of torsion phenomenology in the Solar system.
\end{abstract}

Keywords: Solar system anomalies; Riemann-Cartan spacetime; gravitation models; autoparallel curves; geodesic curves

\section{Introduction}

After one hundred years since its proposal [1], gravitation is still understood in terms of the theory of general relativity (GR). This theory is considered as the pinnacle of classical physics, and the status of its agreement with experiments is very good, although the progress in its verification has been painfully slow [2] due to the weakness of the gravitational interaction and the technical difficulties 
in measuring/observing its predicted effects due to their smallness [3-6]. In the last few years, an important advance has been achieved with the confirmation of the geodetic and the frame-dragging effects upon a gyroscope mounted on an artificial satellite orbiting the Earth. This is known as the Gravity Probe B experiment [7]. With this outstanding result, most of the major deviations from Newtonian gravity, as predicted by GR in the Solar system, are already experimentally checked. Efforts to test a few other ones, such as the Lense-Thirring [8-12] and the post-Newtonian quadrupolar orbital precessions [13,14], are ongoing. Moreover, the discovery of exoplanets orbiting other stars provides an opportunity to obtain additional substantiation of GR [15-17]. In particular, some authors have claimed that the relativistic precession of periastra in exoplanets could be detectable in the near future $[18,19]$. Zhao and Xie have also studied the influence of parametrized post-Newtonian dynamics in their transit times and the possibility of testing GR to a $6 \%$ level [20]. Even testing a putative fifth-force have also been considered [21]. The hot exoplanet WASP-33b also constitutes an excellent natural laboratory for GR [22], because the predicted Lense-Thirring node precession is $3.25 \times 10^{5}$ larger than that of Mercury [23], and this is only one order of magnitude below the measurability threshold for these systems.

On the other hand, since the development of GR in its standard form, there have been many attempts to propose modified theories of gravity capable of predicting new testable phenomena. From the 1920 s to the 1950 s, the main drive of this research was to find a way of unifying gravitation and electromagnetism, although this objective was slowly being abandoned, except for Einstein himself and his collaborators [24]. Many of these theories were characterized by including a nonzero torsion tensor field, as well as the curvature tensor of standard Riemannian geometry. Extended geometries including both curvature and torsion have been used in physics since the early work of Einstein and Cartan [24]. The resulting Einstein-Cartan theory, much later improved by Sciama [25] and Kibble [26], is still considered a viable alternative to standard GR. In fact, it is still actively investigated as attested from the papers, conferences and even books published on this topic [27-29]. The Einstein-Cartan-Sciama-Kibble theory (ECSK) has also an interesting structure, as it can be consistently described as a gauge theory of the Poincaré group [30]. This way, it was put in correspondence with the very successful gauge theory approach to other interactions.

As beautiful as it could be, ECSK theory has not received any experimental support yet. This is not sufficient to dismiss any gravitation theory beforehand, because, as has happened with GR, the experiments are very difficult to design and carry out. Net spin densities are very small in most substances, as alignment of individual atomic spins is random, but it can be large in some elements, such as helium three $\left({ }^{3} \mathrm{He}\right)$ or dysprosium-iron compounds $\left(\mathrm{Dy}_{6} \mathrm{Fe}_{23}\right)$. Ni has suggested to use these elements to build gyroscopes capable of testing PGtheory [31]. Apart from small spin densities, there is also the peculiarity that ECSK predicts null torsion outside macroscopic bodies. The reason for that behavior is that the field equation for torsion relates it linearly with spin density. Consequently, in a vacuum, where spin densities are null, torsion is also zero. Another important application of ECSK theory has been recently found by Popławski, who showed that torsion in the early Universe generates repulsion, and this could solve the flatness and horizon problems without resorting to an ad hoc inflation scenario [32,33]. A class of Poincaré gauge theories with extended Lagrangians quadratic in curvature 
and torsion have also been studied in the last few decades. These theories follow closely the analogy of the gauge paradigms of Weyl and Yang-Mills and, in some cases, predict a propagating torsion [34].

However, exploring alternative theories and models to standard PG is still both viable and timely. Theories in which torsion propagates outside macroscopic bodies can also be developed consistently [35]. As early as 1979, Hojman et al. proposed a model in which torsion is connected with a massless scalar field [36,37]. In this theory, torsion propagates in a vacuum, and torsion waves can be generated by sources with variable spin. At the same time, Hayashi and Shirafuji discussed an alternative to GR in which they revived the notion of Einstein's teleparallelism [38]. In the Hayashi-Shirafuji theory the fundamental entities are the tetrads instead of the metric, and the action is varied with respect to them to obtain the field equations. Interestingly, Hayashi and Shirafuji found a static spherically-symmetric vacuum solution in Weitzenböck spacetime (characterized by a null curvature tensor and a nonzero torsion $[39,40]$ ), which replaces the Schwarzschild solution in standard GR. The so-called new general relativity agrees with the classical tests for light bending, the anomalous advance of the perihelion of Mercury and Shapiro's delay of radar signals [38]. However, this theory fails to predict the geodetic and frame-dragging effects already checked in the Gravity Probe B experiment [7,41].

In 2007, Mao, Tegmark, Guth and Cabi proposed a phenomenological parametrized model for torsion in the Solar system (MTGC model). In this model, the source of torsion is assumed to be the rotational angular momentum of the planets and the Sun [41]. This is not the case in standard PG theory in which only the microscopic spin of elementary particles can generate torsion. The MTGC model does not depend on any specific theoretical framework, as the authors deduce the form of the torsion tensor from symmetry principles as the invariance under rotation, the antisymmetry of the torsion tensor in its covariant indices and the behavior of the angular momentum vector under parity transformations [41]. By using autoparallel or extreme schemes for the spin four vector $S^{\mu}$ or spin fourth tensor $S^{\mu \nu}$, these authors calculate extra contributions to the geodetic and frame-dragging precessions of a gyroscope's spin orbiting around the Earth. They claim that a refined version of Gravity Probe B experiment could be used to determine the values of some combinations of the seven constant parameters used to parametrize torsion. For the time being, error bars in the GPBexperiment are so large that we can only give some estimates on the bounds of the torsion parameters, but compatibility with standard GR is still not excluded by observations. Applying the planetary equations of Lagrange in the Gauss form, March et al. calculated the secular variations of the orbital elements for the planets and the Earth's geodynamics satellites $[42,43]$. In particular, they have found extra precessions rates for the longitude of the ascending node, i.e., an anomalous Lense-Thirring effect, and also a contribution to the precession of the perihelion. Unfortunately, the precision in the determination of the Lense-Thirring effect or the perihelion precession, even for the highly-accurate measurements of the geodynamics satellites, such as LAGEOS, is still not sufficient to evince an irrefutable discrepancy with the predictions of standard GR. It is hoped that the newly-launched LARES [44] satellite may yield an improvement in the accuracy of the ongoing and forthcoming tests of fundamental physics, although also, such a possibility is currently debated [45-48]. Although the authors of these works have given some bounds on the values of the torsion parameters, based on the known error bars from the most recent ephemerides, it is still premature to draw any firm conclusion on the need of modified theories of gravity to explain the data. 
The approaches of Mao et al. [41] and the subsequent spin-off applications by March et al. [42,43] have been heavily criticized by advocates of the PG theory. Hehl et al. have argued the following [49,50]: (i) Postulating that structureless test bodies follow autoparallel trajectories is incorrect in a general relativistic setup. In standard torsion theories test, bodies follow extremal trajectories, as is also the case in GR. The extremal trajectory is derived from the field equations themselves, and this is a theoretical feature of general relativity that should be preserved in any future theory. (ii) The net orbital angular momentum is not an integral over a local density, and consequently, it cannot be the source of torsion in a local field theory of gravity. Concerning the first objection, Kleinert and Pelster argued that in a spacetime with torsion, we must notice that parallelograms are, in general, not closed [51], the closure failure being proportional to the torsion tensor. This implies that the variational principle for finding the extrema of the action must take into account that the variation at the final point is nonzero as a consequence of the closure failure [52]. Using this modified variational principle, Kleinert and Pelster found that the equation of motion of structureless test bodies is given by autoparallel trajectories instead of extremal trajectories [51]. Bel has also shown that an analogy can be established among geodesics in Riemannian spacetime and autoparallels of a Weitzenböck connection [40]. In the absence of torsion extremal and autoparallel trajectories coincides as happens in standard GR. Hehl and Obukhov criticized this approach, because the autoparallel trajectories were not derived from the energy-momentum conservation laws, as is done in the GR and PG theories [50]. However, the closure failure also implies that the energy-momentum tensor of spinless point particles satisfies a different conservation law, as shown by Kleinert [53]. The second objection is, however, lethal to the MTGC model and its consequences. Any consistent theory of gravity must admit only local quantities or quantities obtained as the integration of local densities as sources of the tensor fields.

For these reasons, we investigate in this paper an alternative source for torsion around a rotating sphere. In our model, torsion is related to an axial vector field following the celestial parallels, $\mathbf{A}(r, \theta, \phi)=A(r, \theta, \phi) \hat{\boldsymbol{\phi}}$. This field structure could be obtained from the solution of a local Laplacian equation relating the vector potential with the energy-momentum flux of the rotating body in analogy to the corresponding equation for the magnetic field around a charged rotating sphere. All quantities in this model are local, and the non-locality induced by considering the total angular momentum as the source of torsion is removed. We study the secular evolution of the orbital elements for a test particle orbiting around a rotating central body. Some new effects unknown in GR are found: (i) a secular variation of the semi-major axis of the orbit; and (ii) a secular variation of the orbital eccentricity. As the increase of the astronomical unit is currently being discussed and no conventional explanation has still been found, our model could provide such an explanation, and moreover, we can give estimations on the torsion parameters from the preliminary data on these anomalies [54-56]. If these anomalies are confirmed, torsion fields generated by a circulating potential vector around rotating bodies could provide a parsimonious explanation of these phenomena, and they would stimulate further research in torsion gravity.

The structure of the paper is as follows: In Section 2, we provide a brief review on Riemann-Cartan spacetime as a quick reference for the rest of the paper. Our proposal for the torsion around spherical rotating bodies is discussed in Section 3 by following the symmetry arguments of Mao et al. [41]; autoparallel trajectories and orbital equations for perturbation theory are derived in Section 4. Results for 
the secular variation of the elements and comparison with Solar system anomalies are used in Section 5 to estimate the torsion parameters of our model. The discussion and conclusions are given in Section 6. Appendix A is also included, in which the relation among the perturbing forces in the Sun's and the orbital system of reference is derived.

\section{The Torsion and Contortion Tensors in Riemann-Cartan Spacetime}

In this section, we remind about the main definitions and relations among tensors and the affine connection in Riemann-Cartan spacetime [57]. This spacetime is characterized by a non-zero curvature tensor and a torsion tensor defined as follows:

$$
S_{j k}^{i}=\frac{1}{2}\left(\Gamma_{j k}^{i}-\Gamma_{k j}^{i}\right)
$$

Therefore, a nonvanishing torsion implies that the affine connection is not symmetrical in the two lower indices in contrast with the postulates of ordinary Riemannian geometry. Christoffel's symbols are defined in terms of the metric tensor by the same expression found in Riemannian geometry. However, as we will find below, they do not coincide with the affine connection. Therefore, we have for Christoffel's symbols:

$$
\left\{\begin{array}{c}
i \\
j k
\end{array}\right\}=\frac{1}{2} g^{i l}\left(g_{l k, j}+g_{j l, k}-g_{j k, l}\right),
$$

where the commas denote, as usual, ordinary derivatives with respect to the coordinates. However, covariant and contravariant derivatives of a vector field must be defined in terms of the affine connection by the following relations:

$$
\begin{aligned}
& A_{i \mid j}=A_{i, j}-A_{k} \Gamma_{j i}^{k}, \\
& A_{\mid j}^{i}=A^{i}{ }_{, j}+A^{k} \Gamma_{j k}^{i} .
\end{aligned}
$$

The metric condition is given as usual:

$$
g_{i j \mid k}=g_{i j, k}-g_{h j} \Gamma_{k i}^{h}-g_{i h} \Gamma_{k j}^{h}=0
$$

so the non-metricity is null [41]. By adding up the equivalent equations resulting from Equation (5) by the cyclic permutation of the the three indices, we have:

$$
\left\{\begin{array}{c}
i \\
j k
\end{array}\right\}=\Gamma_{j k}^{i}+K_{j k}^{i},
$$

where $K_{j k}{ }^{i}$ is the contortion tensor defined in terms of the torsion as follows:

$$
\begin{aligned}
K_{j k}^{i} & =-S_{j k}^{i}+g^{i l} g_{h k} S_{j l}^{h}+g^{i l} g_{j h} S_{k l}^{h} \\
K_{j k}^{i} & =-S_{j k}^{i}-S_{j k}^{i}+S_{k j}^{i},
\end{aligned}
$$

where we have used the metric tensor to raise and lower the indices. Similarly, in terms only of covariant indices, we have:

$$
K_{i j k}=S_{j i k}-S_{k i j}+S_{j k i},
$$


from which we deduce the following antisymmetry property:

$$
K_{i j k}=-K_{i k j}
$$

which also implies $K_{i j}{ }^{j}=0$. In the case of the Riemann-Cartan spacetime, we have a generalization of a Ricci identity involving the torsion tensor as follows:

$$
A_{\mid j k}^{i}-A_{\mid k j}^{i}=-A^{h} R_{k j h}{ }^{i}-2 Q_{k j}{ }^{h} A_{\mid h}^{i},
$$

where both the curvature tensor and the torsion tensor appear.

\section{Parametrization of Torsion in Spherically-Symmetric and Axisymmetric Spacetimes}

The main idea of the MTGC model consist of a parametrization of torsion for both the static, spherical and parity symmetric case and the stationary, spherically-axisymmetric spacetime by using dimensional and symmetry arguments [41].

In the first case, we expect torsion to be invariant under the group of spatial rotations, $O(3)$, and, consequently, to involve only invariant quantities, such as the radio vector, $x^{i}, i=1,2,3$, the Kronecker $\delta$-function and the mass of the spherical object generating the field. The most general torsion tensor with these conditions becomes:

$$
\begin{aligned}
S_{0 i}{ }^{0} & =t_{1} \frac{m}{2 r^{3}} x^{i}, \\
S_{j k}^{i} & =t_{2} \frac{m}{2 r^{3}}\left(x^{j} \delta_{k i}-x^{k} \delta_{j i}\right),
\end{aligned}
$$

where $i, j$ and $k$ are spatial indices and $t_{1}, t_{2}$ are functions of $r$ alone to be treated as constants for an orbit of fixed radius. For perturbation calculations, it is highly convenient to transform this result to spherical coordinates by using the identities: $\partial x^{i} / \partial r=\hat{e}_{r}^{i}, \partial x^{i} / \partial \theta=r \hat{e}_{\theta}^{i}$ and $\partial x^{i} / \partial \phi=r \sin \theta \hat{e}_{\phi}^{i}$, which yields for the nonvanishing components:

$$
\begin{aligned}
S_{t r}{ }^{t} & =S_{t i}{ }^{t} \frac{\partial x^{i}}{\partial r}=t_{1} \frac{m}{2 r^{2}} \\
S_{r \theta}{ }^{\theta} & =S_{j k}{ }^{i} \frac{\partial x^{j}}{\partial r} \frac{\partial x^{k}}{\partial \theta} \frac{\partial \theta}{\partial x^{i}}=t_{2} \frac{m}{2 r^{2}}, \\
S_{r \phi}{ }^{\phi} & =S_{r \theta}{ }^{\theta} .
\end{aligned}
$$

Now, we consider the stationary spherically-axisymmetric spacetime whose metric is given, to first order, as:

$$
\begin{aligned}
d s^{2} & =-\left[1-\frac{\rho_{S}}{r}\right] c^{2} d t^{2}+\left[1+\gamma \frac{\rho_{S}}{r}\right] \\
& +r^{2}\left(d \theta^{2}+\sin ^{2} \theta d \phi^{2}\right) \\
& -\left(1+\gamma+\alpha_{1} / 4\right) \frac{\rho_{S} \rho_{J}}{r} c d t d \phi,
\end{aligned}
$$

where $\rho_{S}=2 G M / c^{2}$ is the Schwarzschild radius and $\rho_{J}=J /(M c)$ is a distance given in terms of the total angular momentum of the rotating object, its mass, $M$, and the speed of light, $c$. The constant parameters $\gamma$ and $\alpha_{1}$ are the parametrized post-Newtonian mechanics (PPN) parameters whose values in 
the case of standard general relativity are $\gamma=1, \alpha_{1}=0$ as we will assume in this paper. The non-zero first-order contributions to Christoffel's symbols in Equation (2) are listed below:

$$
\begin{aligned}
& \left\{\begin{array}{c}
t \\
r t
\end{array}\right\}=\left\{\begin{array}{c}
r \\
t t
\end{array}\right\}=\frac{\rho_{S}}{2 r}, \\
& \left\{\begin{array}{c}
t \\
r \phi
\end{array}\right\}=-\frac{3}{2} \sin ^{2} \theta \frac{\rho_{S} \rho_{J}}{r^{2}} \\
& \left\{\begin{array}{c}
r \\
\phi t
\end{array}\right\}=-\sin ^{2} \theta \frac{\rho_{S} \rho_{J}}{2 r^{2}} \\
& \left\{\begin{array}{c}
r \\
r r
\end{array}\right\}=-\frac{\rho_{S}}{2 r^{2}} \\
& \left\{\begin{array}{c}
r \\
\theta \theta
\end{array}\right\}=\rho_{S}-r=\left\{\begin{array}{c}
r \\
\phi \phi
\end{array}\right\} / \sin ^{2} \theta, \\
& \left\{\begin{array}{c}
\theta \\
t \phi
\end{array}\right\}=\frac{\rho_{S} \rho_{J}}{r^{3}} \sin \theta \cos \theta, \\
& \left\{\begin{array}{c}
\theta \\
r \theta
\end{array}\right\}=\left\{\begin{array}{c}
\phi \\
r \phi
\end{array}\right\}=\frac{1}{r}, \\
& \left\{\begin{array}{c}
\theta \\
\phi \phi
\end{array}\right\}=-\sin \theta \cos \theta \text {, } \\
& \left\{\begin{array}{c}
\phi \\
t r
\end{array}\right\}=\frac{\rho_{S} \rho_{J}}{2 r^{4}} \\
& \left\{\begin{array}{c}
\phi \\
t \theta
\end{array}\right\}=-\frac{\cos \theta}{\sin \theta} \frac{\rho_{S} \rho_{J}}{r^{3}} \\
& \left\{\begin{array}{c}
\phi \\
\theta \phi
\end{array}\right\}=\frac{\cos \theta}{\sin \theta},
\end{aligned}
$$

and the corresponding symbols with permutated lower indices, which coincide with the listed ones by symmetry. First-order refers to the fact that we only consider terms proportional to $\rho_{S}, \rho_{J}$ and $\rho_{S} \rho_{J}$ and ignore any higher-order power.

Finally, we will consider the torsion tensor for the stationary spherically-axisymmetric case. Torsion will be associated with an axial vector field $A^{k}$ instead of the angular momentum, as proposed in the MTGC model [41]. This vector field reverses under time reversal and improper rotations. This requires that only those components with a single temporal index are nonvanishing. Moreover, to cancel the minus sign arising in improper rotations, we must include the Levi-Cività symbol, $\epsilon_{i j k}$, because, being a pseudotensor, it also changes sign in improper rotations. With these conditions, it was found that: 


$$
\begin{aligned}
S_{i j}{ }^{t} & =\frac{f_{1}}{2 r^{3}} \epsilon_{i j k} A^{k}+\frac{f_{2}}{2 r^{5}} A^{k} x^{l}\left(\epsilon_{i k l} x^{j}-\epsilon_{j k l} x^{i}\right) \\
& +\frac{f_{6}}{2 r^{5}} A^{k} x_{k} \epsilon_{i j l} x^{l}, \\
S_{t i j} & =\frac{f_{3}}{2 r^{3}} \epsilon_{i j k} A^{k}+\frac{f_{4}}{2 r^{5}} A^{k} x^{l} \epsilon_{i k l} x^{j} \\
& +\frac{f_{5}}{2 r^{5}} A^{k} x^{l} \epsilon_{j k l} x^{i}+\frac{f_{7}}{2 r^{5}} A^{k} x_{k} \epsilon_{i j l} x^{l},
\end{aligned}
$$

where $f_{1}, \ldots, f_{7}$ are constants by dimensional analysis. Notice that Mao et al. [41] identified the vector $A^{k}$ with the angular momentum $J^{k}$, but in the model presented in this paper, it could be a more general vector field. Save for constant prefactors, we chose a vector field with the same structure that the vector potential of a rotating charged sphere is as follows:

$$
\mathbf{A}=\frac{4 G M \Omega R^{2}}{5 c^{3}} \sin \theta\left(-\sin \phi \hat{\mathbf{m}}_{1}+\cos \phi \hat{\mathbf{m}}_{2}\right)
$$

where $\Omega$ is the angular velocity of the rotating central body, $m$ is the mass and $R$ is its radius. The prefactor is, then, proportional to the modulus of the total angular momentum. Notice that we can also write it as $4 G M \Omega R^{2} /\left(5 c^{3}\right)=\rho_{S} \rho_{J}$, where $\rho_{S}=2 G M / c^{2}$ is the Schwarzschild radius of the central body and $\rho_{J}=J /(M c)=2 / 5(\Omega R / c) R$. The unit vector $\hat{\mathbf{m}}_{1}$ points towards the ascending node of the Sun's axial rotation, and $\hat{\mathbf{m}}_{2}$ is perpendicular to it in the equatorial plane of the Sun or the rotating body that we are considering. The nonvanishing components of the torsion tensor corresponding to the static spherically-symmetric case are derived from Equation (13) as given in [41] as follows:

$$
S_{t r}{ }^{t}=t_{1} \frac{m}{2 r^{2}}, S_{r \theta}{ }^{\theta}=S_{r \phi}^{\phi}=t_{2} \frac{m}{2 r^{2}} .
$$

Additionally, those components, opposite in sign, correspond to the permutation of the covariant indices. Similarly, we find from Equations (28) and (29) that additional nonzero components of the torsion tensor for the stationary spherically-axisymmetric case are given by:

$$
\begin{aligned}
& S_{r \theta}{ }^{t}=-S_{\theta r}{ }^{t}=\chi_{1} \frac{\rho_{S} \rho_{J}}{2 r^{2}} \sin \theta, \\
& S_{t \theta}{ }^{r}=-S_{\theta t}{ }^{r}=\chi_{2} \frac{\rho_{S} \rho_{J}}{2 r^{2}} \sin \theta, \\
& S_{t r}^{\theta}=-S_{r t}{ }^{\theta}=\chi_{3} \frac{\rho_{S} \rho_{J}}{2 r^{4}} \sin \theta,
\end{aligned}
$$

where $\chi_{1}, \chi_{2}$ and $\chi_{3}$ are constant parameters. Finally, we must tabulate the values of the nonvanishing components of the contortion tensor (up to first-order in $\rho_{S}, \rho_{J}$ and $\rho_{S} \rho_{J}$ ) by using the relation with the torsion in Equation (7) and Equations (30) and (31). The results are listed below:

$$
\begin{aligned}
& K_{01}{ }^{0}=K_{00}{ }^{1}=-\frac{\rho_{S} t_{1}}{r^{2}}, \\
& K_{22}{ }^{1}=K_{33}{ }^{1} / \sin ^{2} \theta=-\rho_{S} t_{2}, \\
& K_{21}{ }^{2}=K_{31}{ }^{3}=\frac{\rho_{S} t_{2}}{r^{2}}
\end{aligned}
$$




$$
\begin{aligned}
& K_{21}{ }^{0}=\left(\chi_{1}+\chi_{2}+\chi_{3}\right) \frac{\rho_{S} \rho_{J}}{2 r^{2}} \sin \theta, \\
& K_{12}{ }^{0}=\left(\chi_{1}-\chi_{2}-\chi_{3}\right) \frac{\rho_{S} \rho_{J}}{2 r^{2}} \sin \theta, \\
& K_{20}{ }^{1}=\left(\chi_{1}+\chi_{2}+\chi_{3}\right) \frac{\rho_{S} \rho_{J}}{2 r^{2}} \sin \theta, \\
& K_{02}{ }^{1}=\left(\chi_{1}-\chi_{2}+\chi_{3}\right) \frac{\rho_{S} \rho_{J}}{2 r^{2}} \sin \theta, \\
& K_{10}{ }^{2}=\left(\chi_{2}+\chi_{3}-\chi_{1}\right) \frac{\rho_{S} \rho_{J}}{2 r^{4}} \sin \theta, \\
& K_{01}{ }^{2}=\left(\chi_{2}-\chi_{1}-\chi_{3}\right) \frac{\rho_{S} \rho_{J}}{2 r^{4}} \sin \theta .
\end{aligned}
$$

In the next section, we will study the autoparallel trajectories in the spacetime with this contortion tensor. Notice that the torsion and contortion tensor fields are determined by five constant parameters: $t_{1}$, $t_{2}, \chi_{1}, \chi_{2}$ and $\chi_{3}$. The effect of the first two, $t_{1}$ and $t_{2}$, has already been analyzed by March et al. [42,43], and we will be concerned in this paper with the bounds or estimated values of $\chi_{1}, \chi_{2}$ and $\chi_{3}$.

\section{Autoparallel Trajectories and Perturbation Theory}

As in previous models [41-43], we will assume that structureless point particles move along autoparallel trajectories of the Riemann-Cartan spacetime. Therefore, we have that:

$$
\frac{d^{2} x^{\alpha}}{d \tau^{2}}+\left\{\begin{array}{c}
\alpha \\
\mu \nu
\end{array}\right\} \frac{d x^{\mu}}{d \tau} \frac{d x^{\nu}}{d \tau}=K_{\mu \nu}{ }^{\alpha} \frac{d x^{\mu}}{d \tau} \frac{d x^{\nu}}{d \tau}
$$

where $\tau$ is proper time measured along the trajectory. Notice that in a purely Riemannian spacetime, the contortion tensor is null, and Equation (41) is also found for geodesic trajectories. It is usually claimed that only test bodies with a microstructure can couple to torsion [58] and that spinless particles should follow the geodesic trajectories defined by:

$$
\frac{d^{2} x^{\alpha}}{d \tau^{2}}+\left\{\begin{array}{c}
\alpha \\
\mu \nu
\end{array}\right\} \frac{d x^{\mu}}{d \tau} \frac{d x^{\nu}}{d \tau}=0
$$

In standard general relativity, one finds this equation of motion in two ways: as the extremal of the integral of the spacetime element, $d s$, or as a consequence of the field equations, i.e., as the condition that the covariant divergence of the stress-energy tensor has zero value [59]. Both ways are equivalent and lead to the geodesic equation of motion in Equation (42). Mathematically, this coincides with the autoparallels in Equation (41) for zero torsion. If the calculations are translated to a Riemann-Cartan spacetime using holonomic constraints at the initial and final points (with zero variation in these points) of the trajectory, we find again the geodesic trajectories, because only the Christoffel symbols enter into the analysis [53].

However, geodesics are a global concept defined, as they are, as the shortest paths between two points. On the contrary, autoparallels can be defined locally as the straightest paths in spacetime. Therefore, from the fundamental principle of locality in classical field theory, it seems more natural to ascribe physical significance to autoparallels instead of geodesics. 
Kleinert and collaborators [51-53] have studied a new nonholonomic mapping principle from flat spacetime to curved spacetime with torsion in which curvature is described as a disclination and torsion as a dislocation of the spacetime fabric. This implies that a closure failure appears in parallelograms, and the endpoints of a variational trajectory are displaced by:

$$
\delta^{S} b^{\mu}=\delta^{S} q^{\mu}-\delta q^{\mu}
$$

where $\delta^{S}$ denotes the nonholonomic variations and $\delta$ are the auxiliary variations vanishing at the endpoints [53]. Starting from the action:

$$
\mathcal{A}=-\frac{1}{2} \int_{\sigma_{1}}^{\sigma_{2}} d \sigma g_{\mu \nu}(q(\sigma)) \dot{q}^{\mu}(\sigma) \dot{q}^{\nu}(\sigma),
$$

and applying the nonholonomic variations, we get:

$$
\delta^{S} \mathcal{A}=-\int_{\sigma_{1}}^{\sigma_{2}} d \sigma\left(g_{\mu \nu} \dot{q}^{\nu} \delta^{S} \dot{q}^{\mu}+\frac{1}{2} \partial_{\mu} g_{\lambda \kappa} \delta^{S} q^{\mu} \dot{q}^{\lambda} \dot{q}^{\kappa}\right),
$$

where the dot denotes a derivative with respect the proper time, $\sigma$. In terms of the multivalued tetrads $e^{i}{ }_{\nu}$, $i=0, \ldots, 3, \mu=0, \ldots, 3$, the metric tensor is defined as follows $g_{\mu \nu}=e_{\mu}^{i} e_{\nu}^{i}$, and the affine connection is given by $\Gamma_{\mu \nu}{ }^{\lambda}=e_{i}^{\lambda} \partial_{\mu} e_{\nu}^{i}$. Taking also into account that $\partial_{\mu} g_{\nu \lambda}=\Gamma_{\mu \nu \lambda}+\Gamma_{\mu \lambda \nu}$ and integrating by parts, we obtain:

$$
\begin{aligned}
\delta^{S} \mathcal{A} & =-\int_{\sigma_{1}}^{\sigma_{2}} d \sigma\left[-g_{\mu \nu}\left(\ddot{q}^{\nu}+\left\{\begin{array}{c}
\nu \\
\lambda \kappa
\end{array}\right\} \dot{q}^{\lambda} \dot{q}^{\kappa}\right) \delta q^{\mu}\right. \\
& \left.+\left(g_{\mu \nu} \dot{q}^{\nu} \frac{d}{d \sigma} \delta^{S} b^{\mu}+\Gamma_{\mu \lambda \kappa} \delta^{S} b^{\mu} \dot{q}^{\lambda} \dot{q}^{\kappa}\right)\right]
\end{aligned}
$$

By using the differential equation for the nonholonomic variation $\delta^{S} b^{\mu}$ :

$$
\frac{d}{d \sigma} \delta^{S} b^{\mu}=-\Gamma_{\lambda \nu}^{\mu} \delta^{S} b^{\lambda} \dot{q}^{\nu}+2 S_{\lambda \nu}{ }^{\mu} \dot{q}^{\lambda} \delta q^{\nu}
$$

Kleinert finds:

$$
\delta^{S} \mathcal{A}=\int_{\sigma_{1}}^{\sigma_{2}} d \sigma g_{\mu \nu}\left(\ddot{q}^{\nu}+\Gamma_{\lambda \kappa}^{\nu} \dot{q}^{\lambda} \dot{q}^{\kappa}\right) \delta q^{\mu}=0 .
$$

As the auxiliary variations are arbitrary, but null at the endpoints, $\sigma_{1}$ and $\sigma_{2}$, we get from this the autoparallel equation instead of the geodesic. A similar derivation was discussed also by Kleinert and Pelster [51]. Further details are also given in several textbooks [60,61]. Autoparallels also satisfy a gauge invariance relating the autoparallel equations of motion in different Riemann-Cartan spacetimes [62]. This property could find a deeper physical meaning, apart from its mathematical interest, in future theories of gravity involving curvature and torsion.

The question of the relevance for the physics of the standard method or the method based on nonholonomic variations for the derivation of the equations of motion in Riemann-Cartan spacetime should be decided experimentally if, as we suggest, there is an additional torsion structure in spacetime. This question cannot be answered in the context of standard general relativity, because, in this case, autoparallel and geodesic equations coincide.

In Riemann-Cartan spacetime, the right-hand side of Equation (41) represents a perturbing force whose effects can be calculated by standard perturbation theory in celestial mechanics. We should 
calculate the first-order perturbation terms arising from the contortion tensor field in Equations (32)-(40). As the planets in the Solar system move with velocities much smaller than the speed of light, we can identify the proper time with the ephemeris time or the atomic time used by astronomers, i.e., $\tau=t$. We will also consider that the torsion parameters $t_{1}=t_{2}=0$, because it has been shown that $t_{1}$ can be absorbed in a redefinition of the mass of the source, and $t_{2}$ does not appear in the equations of the trajectories $[42,43]$.

The components of the perturbing torsion terms are then given by:

$$
\delta F^{i}=K_{\mu \nu}{ }^{i} \frac{d x^{\mu}}{d \tau} \frac{d x^{\nu}}{d \tau},
$$

with $i=1,2,3$ corresponding to the radial, polar and azimuthal coordinates, respectively. The leading term of the radial perturbing acceleration is found by direct substitution of Equations (32)-(40) into Equation (49):

$$
\begin{aligned}
\delta a_{r} & =\delta F^{r}=c\left(K_{02}{ }^{1}+K_{20}{ }^{1}\right) \dot{\theta} \\
& =c\left(\chi_{1}+\chi_{3}\right) \frac{\rho_{S} \rho_{J}}{r^{2}} \sin \theta \dot{\theta},
\end{aligned}
$$

ignoring corrections $\mathcal{O}\left(\dot{\theta}^{2}\right)$ and $\mathcal{O}\left(\dot{\phi}^{2}\right)$, $c$ being the speed of light in a vacuum, $d x^{0} / d t=c$ and $\dot{\theta}=d \theta / d t$. Similarly, for the polar component of the perturbing force, we find:

$$
\begin{aligned}
& \delta a_{\theta}=r \delta F^{\theta}=r\left(K_{01}{ }^{2}+K_{10}{ }^{2}\right) \dot{r} \\
& =c\left(\chi_{2}-\chi_{1}\right) \frac{\rho_{S} \rho_{J}}{r^{3}} \sin \theta \dot{r} \text {. }
\end{aligned}
$$

On the other hand, the azimuthal component of the perturbing torsion force is zero, as expected for an axisymmetric source, $\delta a_{\phi}=0$. The perturbing accelerations in Equations (50) and (51) are corrections to the accelerations in spherical coordinates in the system of reference of the Sun (see Appendix 6), which are obtained from the left-hand side of the equations for the autoparallel trajectories and the first-order Christoffel symbols in Equations (17)-(27) as follows:

$$
\begin{aligned}
& a_{r}=\ddot{r}-r \dot{\theta}^{2}-r \dot{\phi}^{2} \sin ^{2} \theta, \\
& a_{\theta}=r \ddot{\theta}+2 \dot{r} \dot{\theta}-r \dot{\phi}^{2} \sin \theta \cos \theta, \\
& a_{\phi}=r \ddot{\phi} \sin \theta+2 \dot{r} \dot{\phi} \sin \theta+2 r \dot{\theta} \dot{\phi} \cos \theta .
\end{aligned}
$$

As our objective is to apply celestial mechanics perturbation techniques, we will find it convenient to calculate the radial, $\mathcal{R}$, tangential to the orbit, $\mathcal{T}$, and normal to the orbital plane, $\mathcal{N}$, components of the perturbing torsion force per unit mass. In Equations (88)-(90) in the Appendix 6, we have found those components in terms of the accelerations in spherical coordinates in a system of reference whose $z$ axis is the rotation axis of the Sun. The relation is expressed in terms of a transformation matrix $\alpha_{i j}$, $i, j=1,2,3$ obtained as the set of scalar products among the vectors in the orbital system of reference and the Sun's system of reference, as given in Equation (79). 
Direct substitution of Equations (50) and (51) and $\delta a_{\phi}=0$ into Equations (88)-(90) yields:

$$
\begin{aligned}
\mathcal{R}= & c\left(\chi_{1}+\chi_{3}\right) \frac{\rho_{S} \rho_{J}}{r^{2}}\left(\alpha_{13} \sin \nu\right. \\
- & \left.\alpha_{23} \cos \nu\right) \dot{\nu} \\
\mathcal{T}= & c\left(\chi_{2}-\chi_{1}\right) \frac{\rho_{S} \rho_{J}}{r^{3}}\left(\alpha_{13} \sin \nu\right. \\
- & \left.\alpha_{23} \cos \nu\right) \dot{r} \\
\mathcal{N}= & c\left(\chi_{2}-\chi_{1}\right) \frac{\rho_{S} \rho_{J}}{r^{3}} \sin \theta \\
& \left(\alpha_{31} \cos \theta \cos \phi+\alpha_{32} \cos \theta \sin \phi-\alpha_{33} \sin \theta\right) \dot{r} \\
= & c \alpha_{33}\left(\chi_{1}-\chi_{2}\right) \frac{\rho_{S} \rho_{J}}{r^{3}} \dot{r} .
\end{aligned}
$$

Notice that all terms in Equations (55)-(57) are constants or can be written in terms of the true anomaly. The relations among the polar, $\theta$, and azimuthal, $\phi$, angles and the true anomaly, $\nu$, are given in Equations (80)-(82). Using these relations and the identity in Equation (83), we have found the simplifications for $\mathcal{T}$ and $\mathcal{N}$.

For the radio vector and radial velocity, we have [63-65]:

$$
\begin{aligned}
r & =\frac{p}{1+\epsilon \cos (\nu-\omega)}, \\
\dot{r} & =\sqrt{\frac{\mu}{p}} \epsilon \sin (\nu-\omega),
\end{aligned}
$$

where $p=a\left(1-\epsilon^{2}\right)$ is the semilatus rectum, $\epsilon$ is the eccentricity, $a$ is the semimajor axis, $\omega$ is the argument of the perihelion and $\mu=G M$ is the product of the gravitational constant and the mass of the Sun. The relation among time, $t$, and the true anomaly, $\nu$, will also be useful in the following perturbation calculations:

$$
d t=\frac{T}{2 \pi} \frac{\left(1-\epsilon^{2}\right)^{3 / 2}}{(1+\epsilon \cos (\nu-\omega))^{2}} d \nu,
$$

where the orbital period, $T$, is given by Kepler's third law: $T=2 \pi a^{3 / 2} / \mu^{1 / 2}$. We are now ready for calculating the perturbations in the orbital elements as a consequence of the torsion force in Equations (55)-(57). Following the classical treatment of Burns [65], we can write for the semimajor axis:

$$
\frac{\dot{a}}{a}=\frac{2 a}{\mu} \dot{E}=\frac{2 a}{\mu}(\dot{r} \mathcal{R}+r \dot{\nu} \mathcal{T})
$$

where $E$ is the total energy. After some simplifications using Equations (55), (56), (58) and (59), we have:

$$
\begin{aligned}
d a= & \frac{c T}{\pi} \frac{\rho_{S} \rho_{J}}{a^{2}} \frac{\epsilon}{\left(1-\epsilon^{2}\right)^{5 / 2}}\left(\chi_{2}+\chi_{3}\right) \sin (\nu-\omega) \\
& (1+\epsilon \cos (\nu-\omega))^{2}\left(\alpha_{13} \sin \nu-\alpha_{23} \cos \nu\right) d \nu .
\end{aligned}
$$

For the instantaneous variation of the eccentricity, we start from:

$$
\dot{\epsilon}=\frac{\epsilon^{2}-1}{2 \epsilon}\left[-\frac{\dot{a}}{a}+\frac{2 r \mathcal{T}}{H}\right],
$$


where $H=\sqrt{\mu p}=\sqrt{G M a\left(1-\epsilon^{2}\right)}$ is the angular momentum per unit mass. By using Equations (61), (56) and (59) conjointly with Equation (62), we arrive at:

$$
\begin{aligned}
d \epsilon= & \frac{c T}{2 \pi a} \frac{\rho_{S} \rho_{J}}{a^{2}} \frac{\sin (\nu-\omega)}{\left(1-\epsilon^{2}\right)^{1 / 2}}\left(\alpha_{13} \sin \nu-\alpha_{23} \cos \nu\right) \\
& {\left[\frac{\left(\chi_{2}+\chi_{3}\right)}{1-\epsilon^{2}}(1+\epsilon \cos (\nu-\omega))^{2}+\chi_{1}-\chi_{2}\right] d \nu . }
\end{aligned}
$$

Torsion also induces an extra precession of the longitude of the ascending node of the planetary orbits in addition to the Lense-Thirring effect arising in standard general relativity. This precession rate is proportional to the normal component to the planetary orbits of the perturbing force, as found in perturbation theory [63]:

$$
\frac{d \Omega}{d t}=\frac{r \mathcal{N} \sin \nu}{H \sin I}=\sqrt{\frac{p}{G M}} \frac{\mathcal{N}}{\sin I} \frac{\sin \nu}{1+\epsilon \cos (\nu-\omega)},
$$

where $I$ is the orbital inclination and $p=a\left(1-\epsilon^{2}\right)$ is the semi-latus rectum. From the expression of the normal component of the perturbing force in Equation (57) and the contribution to the precession of the longitude of the ascending node in Equation (64), we obtain:

$$
d \Omega=\alpha_{33}\left(\chi_{1}-\chi_{2}\right) \frac{c T}{2 \pi a} \frac{\rho_{S} \rho_{J}}{a^{2}} \frac{\epsilon}{\left(1-\epsilon^{2}\right)^{3 / 2}} \frac{\sin \nu}{\sin I} \sin (\nu-\omega) d \nu .
$$

Finally, for the extra precession of the perihelion contributed by spacetime torsion, we find:

$$
\begin{aligned}
& d \omega=\left(\chi_{2}-\chi_{1}\right) \frac{c T}{2 \pi a} \frac{\rho_{S} \rho_{J}}{a^{2}} \frac{1}{\left(1-\epsilon^{2}\right)^{3 / 2}} \\
& \left(\sin ^{2}(\nu-\omega) \frac{2+\epsilon \cos (\nu-\omega)}{(1+\epsilon \cos (\nu-\omega))^{3}}\left(\alpha_{13} \sin \nu-\alpha_{23} \cos \nu\right)\right. \\
& \left.+\epsilon \alpha_{33} \cot I \sin \nu \sin (\nu-\omega)\right) d \nu \\
& -c\left(\chi_{1}+\chi_{3}\right) \sqrt{\frac{a}{G M}} \frac{\rho_{S} \rho_{J}}{a^{2}} \frac{1}{\epsilon\left(1-\epsilon^{2}\right)^{3 / 2}} \\
& \cos (\nu-\omega)\left(\alpha_{13} \sin \nu-\alpha_{23} \cos \nu\right) d \nu
\end{aligned}
$$

In the next section, we will discuss the predictions of Equations (61)-(66) for the variation of the orbital elements of the planet and its possible connection with certain anomalies recently found by astronomers.

\section{Results}

As we cannot give to the parameters $\chi_{1}, \chi_{2}$ and $\chi_{3}$ definite values on a theoretical basis, it is not possible, in principle, to make predictions on the variation of the orbital elements as a consequence of torsion in our model. It is reasonable to assume that these parameters are of the order of unity, if there is a theory consistent with the torsion model, but this is not sufficient to suggest any reliable prediction. However, we can use an inductive approach by assuming that some anomalies recently found for the planetary orbits are the consequence of torsion gravity arising in a theory that includes the phenomenological model discussed in this paper as a particular case. Specifically, we refer to the 
anomalous secular increase of the astronomical unit (AU) first reported by Krasinsky and Brumberg in 2004 [66]. The analysis of databases of radar and laser ranging and spacecraft observations in the last few decades showed that the astronomical unit increases by $15 \pm 4$ meters per century. An independent study by Standish reduced this figure to $7 \pm 2$ meters per century [67]. This problem has even motivated the International Astronomical Union to redefine the $\mathrm{AU}$ as a constant and, as a consequence, to remove the Gaussian constant of mass from the list of astronomical constants [56]. However, these redefinitions of constants do not solve the problem pointed out by the aforementioned astronomers. However, we should notice that, according to this new definition, it is not rigorous to compare the rates of the semi-major axes of the planets with the rates of the astronomical unit, because this is fixed. On the other hand, we can use the recent reports on a secular decrease of the mass parameter $\mu=G M$ of the Sun [56]. The average of the rates determined with EPM2008, EPM2010 and EPM2011 ephemerides is $\mu=(5.73 \pm 4.27) \times 10^{-14} \mathrm{yr}^{-1}$. It is known from the perturbation analysis of the planetary orbits in a scenario of a diminishing gravitational constant that the semi-major axis increase as [68]:

$$
\frac{\dot{a}}{a}=-\frac{\dot{\mu}}{\mu}
$$

which implies a rate of $0.86 \pm 0.64$ meters per century. It is important to point out that the recent analyses with the INPOP13cephemerides are statistically compatible with a zero variation of the Sun's mass parameter [56]. Some conventional and unconventional attempts for an explanation of the variation rates for the semi-major axes of the planets have been suggested, but there is still no convincing solution of the problem [69-73].

It is interesting to notice that Equation (61) implies a variation of the semimajor orbital axes for $\eta=\chi_{2}+\chi_{3} \neq 0$. Moreover, if we assume that:

$$
\chi_{1}+\chi_{3}=0
$$

it is found from Equations (61)-(66) that the variations of $a, \epsilon, \Omega$ and $\omega$ depend only on the single parameter $\eta=\chi_{2}-\chi_{1}$. We will choose the condition in Equation (68) without losing the perspective that other possibilities are compatible with our model, even the case in which no secular change is found for the semimajor planetary axes, i.e., the case $\eta=0$.

Using this condition and averaging Equations (61), (63), (65) and (66) over a whole orbit, we obtain the following results for the variation of the elements in one year:

$$
\begin{aligned}
\frac{\Delta a}{a}= & \eta \frac{\operatorname{Ly}}{a} \frac{\Omega R}{c}\left(\frac{R}{a}\right)^{2} \frac{\epsilon}{\left(1-\epsilon^{2}\right)^{5 / 2}} \\
& \left(1+\frac{\epsilon^{2}}{4}\right)\left(\alpha_{13} \cos \omega+\alpha_{23} \sin \omega\right), \\
\Delta \epsilon= & \eta \frac{5 \operatorname{Ly}}{8 a} \frac{\rho_{S} \rho_{J}}{a^{2}} \frac{\epsilon^{2}}{\left(1-\epsilon^{2}\right)^{3 / 2}} \\
& \left(\alpha_{13} \cos \omega+\alpha_{23} \sin \omega\right), \\
\Delta \Omega= & -\eta \alpha_{33} \frac{\operatorname{Ly}}{2 a} \frac{\rho_{S} \rho_{J}}{a^{2}} \frac{\epsilon}{\left(1-\epsilon^{2}\right)^{3 / 2}} \frac{\cos \omega}{\sin I},
\end{aligned}
$$




$$
\begin{aligned}
\Delta \omega= & \eta \frac{\mathrm{Ly}}{2 a} \frac{\rho_{S} \rho_{J}}{a^{2}} \frac{\epsilon}{\left(1-\epsilon^{2}\right)^{3 / 2}} \\
& {\left[\alpha_{33} \cot I \cos \omega-\frac{5}{4}\left(\alpha_{13} \sin \omega-\alpha_{23} \cos \omega\right)\right], }
\end{aligned}
$$

where Ly stands for a light year. By assuming that the increase of the astronomical unit is obtained as an average over the inner planets, Mercury, Venus, the Earth and Mars, and taking the value reported by Standish, $\Delta \mathrm{AU}=7 \pm 2$ meters per century, we find that $\eta=-0.154$. If we take the values deduced from the variation of the mass parameter $G M$ of the Sun as reported in the EPM2008-2011 ephemerides, a smaller value $\eta=-0.019$ is found. In these calculation, the elements of the planets as given in $[74,75]$ were used. We can also make some predictions for the variation of the other orbital elements and check if they are consistent with the presented observations. We should see that, at least, our model is not inconsistent in this sense.

For the secular variation of the eccentricity for this value of $\eta$, we find $\Delta \epsilon=4.57 \times 10^{-13}$ in one year in the case of Mercury and even lower values for the other planets. This is below the precision threshold for present determinations of this magnitude [69].

Possible anomalous contributions to the secular node precessions are currently considered in recent ephemerides. For the moment, no statistically-significant results have been obtained with the attained precisions, but these values set an upper limit to any prediction by theoretical models. For INPOP10a, these corrections are listed in milliarcseconds per century and compared to our predictions from Equation (71) in Table 1. The most recent ephemerides, INPOP10a and EPM2011, in connection with possible Solar system anomalies have been discussed by Iorio [56].

Table 1. Corrections to the secular node precessions as obtained in the INPOP10a ephemeris and predictions of the torsion gravity model discussed in this paper in milliarcseconds per century.

\begin{tabular}{ccc}
\hline Planet & $\boldsymbol{\Delta} \dot{\boldsymbol{\Omega}}($ INPOP10a) & $\boldsymbol{\Delta} \dot{\boldsymbol{\Omega}}$ (Torsion Gravity) \\
\hline Mercury & $1.4 \pm 1.8$ & 0.30 \\
Venus & $0.2 \pm 1.5$ & $2.14 \times 10^{-2}$ \\
Earth & $0.0 \pm 0.9$ & $-1.70 \times 10^{-4}$ \\
Mars & $-0.05 \pm 0.13$ & $2.62 \times 10^{-3}$ \\
Jupiter & $-40 \pm 42$ & $1.51 \times 10^{-5}$ \\
Saturn & $-0.1 \pm 0.4$ & $1.31 \times 10^{-5}$ \\
\hline
\end{tabular}

The corrections to the standard secular perihelion precessions are given in Table 2 and compared to the predictions of our torsion gravity model.

Inspection of Tables 1 and 2 shows that the predictions of the model are compatible with both ephemerides at the $2 \sigma$ level. Therefore, in the context of the torsion gravity model, the anomalous increase of the astronomical unit is consistent with the rest of measurements on possible corrections to other orbital elements. On the other hand, we have shown that only for Mercury, it seems that an 
improved ephemeris could detect a statistically-significant nonzero correction for $\Delta \dot{\Omega}$ and $\Delta \dot{\omega}$ in the foreseeable future.

Table 2. Corrections to the secular perihelion precessions as obtained in the INPOP10a and EPM2011ephemerides and predictions of the torsion gravity model discussed in this paper in milliarcseconds per century.

\begin{tabular}{cccc}
\hline Planet & $\boldsymbol{\Delta} \dot{\boldsymbol{\omega}}($ INPOP10a) & $\boldsymbol{\Delta} \dot{\boldsymbol{\omega}}($ EPM2011) & $\boldsymbol{\Delta} \dot{\boldsymbol{\omega}}$ (Torsion Gravity) \\
\hline Mercury & $0.4 \pm 0.6$ & $-2.0 \pm 3.0$ & -0.622 \\
Venus & $0.2 \pm 1.5$ & $2.6 \pm 1.6$ & $-4.28 \times 10^{-3}$ \\
Earth & $-0.2 \pm 0.9$ & $0.19 \pm 0.19$ & $-1.03 \times 10^{-4}$ \\
Mars & $-0.04 \pm 0.15$ & $-0.02 \pm 0.037$ & $-4.9 \times 10^{-3}$ \\
Jupiter & $-41 \pm 42$ & $58.7 \pm 28.3$ & $-3.48 \times 10^{-5}$ \\
Saturn & $0.15 \pm 0.65$ & $-0.32 \pm 0.47$ & $-2.69 \times 10^{-5}$ \\
\hline
\end{tabular}

\section{Conclusions}

The advancement of physics can only proceed by a continuous interplay between theory and experiment. This healthy interaction allows for a selection of the most promising hypotheses among the different proposals. Gravity theory has been an exception to this methodological rule for the most part of the 20th century, because experiments are very difficult to develop, as they usually imply very accurate devices, and these must be set into orbit to perform the measurements [7,8]. Gravity being the weakest of all interactions is also the one we know least, because accurate tests of all general relativity predictions are still lacking [2]. For example, Lense-Thirring precession of orbital nodes is only known with a wide error bar from the laser range monitoring of the geodynamic satellites [8-10,76-78]. A further, non-negligible source of difficulty in gravitational experiments resides in the extremely long times required either to collect data or to analyze them: the Gravity Probe B is a case-study example [7].

The experimental situation has been complicated in recent years because of the discovery of a set of anomalies that, apparently, cannot be explained conventionally (see, e.g., the recent review by Iorio [56]). Similarly, the most recent ephemerides has allowed the determination of upper bounds on the variation of the orbital elements of the planets beyond the predictions of classical perturbation theory and general relativity [56,75]. Although some of these anomalies may lose their statistical significance in the more or less near future in view of further observations and related analyses, it is nonetheless important to discuss the possibility that they may constrain theories and extensions beyond general relativity.

Many extensions of general relativity, some of them proposed by Einstein himself, have made use of the concept of torsion [24]. These ideas coalesced in the 1960s and 1970s in the so-called Einstein-Cartan-Sciama-Kibble (ECSK) theory in which torsion is connected with the microscopic spin density and does not propagate outside massive bodies [30]. This theory is still considered a viable alternative to general relativity, but it suffers from a total lack of experimental support, despite some claims that it could explain inflation [32,33]. This situation leaves room for the study of more alternatives without restricting to a given mathematical formalism. In such a spirit, Mao et al. proposed in 2007 the 
MTGC parametrized model in which torsion is connected to macroscopic angular momentum [41]. It was shown that this model can be constrained by perturbations in the orbital elements of the planets and geodynamic satellites $[42,43]$.

Hehl et al. [49,50] have pointed out that it is inconsistent to use total angular momentum, i.e., a quantity not obtained by integration over local densities, as the source of a local field quantity, such as torsion. To avoid this inconsistency, we have modified the approach of the original MTGC model by connecting torsion with a local circulating vector potential as the one obtained in classical electromagnetism for a rotating charged sphere. This way, we have shown that a new phenomenon, qualitatively distinct from those obtained in general relativity, is predicted. Namely, a secular increase of the semi-major axes of the planets [66,67]. This problem has attracted the attention of several authors in recent years, who have tried to find explanations in terms of nonstandard and conventional hypotheses [56,69,70,72]. This observation remains unexplained and, although it could be dismissed by more precise analyses in the future, it deserves further attention. We have shown that our torsion model is compatible with these observations for a value of the parameters of the order of unity.

Moreover, planetary ephemerides are becoming increasingly precise year after year. It is still premature to state that statistically-significant anomalies have been revealed in the secular precessions of the longitude of the ascending node and the argument of the perihelion, but the uncertainty intervals are promisingly small [56]. Anyway, it is now clear that any deviation from the predictions of general relativity (once we take into account standard perihelion precessions and the gravitomagnetic Lense-Thirring effect) is expected only in the range of a few milliarcseconds per century.

We have also shown that extra secular precessions of these elements are the consequence of torsion, but for any planet, they are very small in relation to the confidence intervals of the INPOP10a and EPM2011 ephemerides. However, for the case of Mercury, they could be detected in the foreseeable future, because they lie in the range of a few tenths of milliarcseconds per century. The important fact is that this agreement is achieved in consistency with an increase of the astronomical unit of a few meters per century. This means that testing a torsion gravity extension of general relativity as the one discussed in this paper is within the reach of modern observation techniques and data analyses in astronomy.

We conclude that further experiments and observations are required, achieving the maximum precision possible with present-day technology, to confirm or dismiss possible anomalies beyond general relativity in the secular evolution of the elements of the planets and spacecraft. From these future observations, the model proposed in this paper could receive further support. In such a case, it could serve as the basis for a consistent theory of torsion gravity obtained by the scientific method of induction from experience.

\section{Acknowledgments}

The author gratefully acknowledges Lluís Bel for many useful comments and discussions.

\section{Conflicts of Interest}

The author declares no conflict of interest. 


\section{Appendix: Relation among the Sun's System of Reference and the Orbital Coordinates}

Firstly, we define the ecliptic system of reference: $\hat{k}$ is a unit vector perpendicular to the ecliptic plane; $\hat{\imath}$ points towards the point of Aries; and $\hat{\jmath}$ is perpendicular to the preceding unit vectors in such a way that we have a right-handed Cartesian coordinate system [75].

The orbital plane of a given planet is then characterized by two angles: the inclination $\iota$ with respect to the ecliptic plane and the angle $\Omega$ among the line of nodes (i.e., the intersection among the two planes) and the point of Aries. Consequently, we can write the unit vector of the orbital system of reference as follows:

$$
\begin{aligned}
& \hat{\mathbf{n}}_{1}=\cos \Omega \hat{\boldsymbol{\imath}}+\sin \Omega \hat{\boldsymbol{\jmath}} \\
& \hat{\mathbf{n}}_{2}=-\cos \iota \sin \Omega \hat{\imath}+\cos \iota \cos \Omega \hat{\boldsymbol{\jmath}}+\sin \iota \hat{\boldsymbol{k}} \\
& \hat{\mathbf{n}}_{3}=\sin \iota \sin \Omega \hat{\imath}-\sin \iota \cos \Omega \hat{\boldsymbol{\jmath}}+\cos \iota \hat{\boldsymbol{k}}
\end{aligned}
$$

Similarly, we can define the inclination of the Sun's axis and the longitude of the ascending node of its equator. These two angles determine the orientation of the Sun's rotation axis on space and were obtained in the 19th century by careful observations of Carrington. Carrington's elements, as they are called, are given by [79,80]:

$$
\begin{aligned}
\iota_{c} & =7.25^{\circ}, \\
\Omega_{c} & =73.67^{\circ}+0.013958^{\circ}(t-1850),
\end{aligned}
$$

where $t$ is the year of observation. We define the Sun's system of reference as the Cartesian system obtained by the three unit vectors $\hat{\mathbf{m}}_{i}, i=1,2,3$ whose expression in terms of the Carrington elements is also given by Equation (73).

The planet's orbital radius vector is usually written as $[63,64]$ :

$$
\mathbf{r}=p \frac{\cos \nu \hat{\mathbf{n}}_{1}+\sin \nu \hat{\mathbf{n}}_{2}}{1+\epsilon \cos (\nu-\omega)},
$$

where $p=a\left(1-\epsilon^{2}\right)$ is the semilatus rectum, $a$ the semi-major axis, $\epsilon$ the orbital eccentricity, $\nu$ is the true anomaly and $\omega$ is the argument of the perihelion. Notice that we use an unconventional definition of the true anomaly as the angle among the radius vector and the ascending node of the planet instead of measuring it from the perihelion as usual.

We now introduce the transformation matrix $\alpha_{i j}$ as the scalar products of the unit vectors of the orbital and the Sun's system of reference:

$$
\alpha_{i j}=\hat{\mathbf{n}}_{i} \cdot \hat{\mathbf{m}}_{j}, \quad i, j=1,2,3 .
$$

If $\theta, \phi$ are, respectively, the polar angle and azimuthal angle in the Sun's system of reference, we find from Equation (78) and using the definition in Equation (79) the relation among them and the true anomaly, $\nu$, in the following form: 


$$
\begin{aligned}
\cos \theta & =\frac{\mathbf{r}}{r} \cdot \hat{\mathbf{m}}_{3}=\alpha_{13} \cos \nu+\alpha_{23} \sin \nu \\
\sin \theta \cos \phi & =\frac{\mathbf{r}}{r} \cdot \hat{\mathbf{m}}_{1}=\alpha_{11} \cos \nu+\alpha_{21} \sin \nu \\
\sin \theta \cos \phi & =\frac{\mathbf{r}}{r} \cdot \hat{\mathbf{m}}_{2} \\
& =\alpha_{12} \cos \nu+\alpha_{22} \sin \nu .
\end{aligned}
$$

The coefficients of the transformation matrix satisfy some useful identities:

$$
\sum_{k=1}^{3} \alpha_{i k} \alpha_{j k}=\delta_{i j}, i, j=1,2,3,
$$

where $\delta_{i j}$ is Kronecker's delta. The spherical unit vectors in the Sun's system of reference are given as:

$$
\begin{aligned}
\hat{\mathbf{r}} & =\sin \theta \cos \phi \hat{\mathbf{m}}_{1} \\
& +\sin \theta \cos \phi \hat{\mathbf{m}}_{2}+\cos \theta \hat{\mathbf{m}}_{3} \\
\hat{\boldsymbol{\theta}} & =\cos \theta \cos \phi \hat{\mathbf{m}}_{1} \\
& +\sin \theta \sin \phi \hat{\mathbf{m}}_{2}-\sin \theta \hat{\mathbf{m}}_{3} \\
\hat{\boldsymbol{\phi}} & =-\sin \phi \hat{\mathbf{m}}_{1}+\cos \phi \hat{\mathbf{m}}_{2} .
\end{aligned}
$$

From Equations (80) and (84), we can also find the tangential unit vector to the planetary orbit:

$$
\begin{aligned}
\hat{\boldsymbol{\nu}}=\frac{d \hat{\mathbf{r}}}{d \nu} & =\left(-\alpha_{11} \sin \nu+\alpha_{21} \cos \nu\right) \hat{\mathbf{m}}_{1} \\
& +\left(-\alpha_{12} \sin \nu+\alpha_{22} \cos \nu\right) \hat{\mathbf{m}}_{2} \\
& +\left(-\alpha_{13} \sin \nu+\alpha_{23} \cos \nu\right) \hat{\mathbf{m}}_{3}
\end{aligned}
$$

Orthogonality with $\hat{\mathbf{r}}$ and normalization to the unity modulus can be shown by applying the identities in Equation (83).

Now, we can find the radial, $\mathcal{R}$, tangential to the orbit, $\mathcal{T}$, and normal to the orbital plane, $\mathcal{N}$, components of the perturbing force per unit mass in terms of the accelerations, $a_{r}, a_{\theta}$ and $a_{\phi}$, in the Sun's spherical system of reference. It is obvious that:

$$
\mathcal{R}=a_{r}
$$

For the tangential component, we have:

$$
\begin{aligned}
\mathcal{T} & =\left(a_{\theta} \hat{\boldsymbol{\theta}}+a_{\phi} \hat{\boldsymbol{\phi}}\right) \cdot \hat{\boldsymbol{\nu}} \\
& =\frac{a_{\theta}}{\sin \theta}\left(\alpha_{13} \sin \nu-\alpha_{23} \cos \nu\right) \\
& +\frac{a_{\phi}}{\sin \theta}\left(\alpha_{11} \alpha_{22}-\alpha_{12} \alpha_{21}\right)
\end{aligned}
$$


after some simplifications using Equations (80)-(87). Finally, for the normal component, we find:

$$
\begin{aligned}
\mathcal{N} & =\left(a_{\theta} \hat{\boldsymbol{\theta}}+a_{\phi} \hat{\boldsymbol{\phi}}\right) \cdot \hat{\mathbf{n}}_{3} \\
& =a_{\theta}\left(\alpha_{31} \cos \theta \cos \phi+\alpha_{32} \cos \theta \sin \phi-\alpha_{33} \sin \theta\right) \\
& +a_{\phi}\left(-\alpha_{31} \sin \phi+\alpha_{32} \cos \phi\right)
\end{aligned}
$$

Notice that the angles $\theta$ and $\phi$ can be formally expressed in terms of the true anomaly by using Equation (80).

\section{References}

1. Iorio, L. Editorial for the Special Issue 100 Years of Chronogeometrodynamics: The Status of the Einstein's Theory of Gravitation in Its Centenial Year. Universe 2015, 1, 38-81.

2. Will, C.M. The Confrontation between General Relativity and Experiment. Living Rev. Relativ. 2006, 9,3 .

3. Lämmerzahl, C.; Ciufolini, I.; Dittus, H.; Iorio, L.; Müller, H.; Peters, A.; Samain, E.; Scheithauer, S.; Schiller, S. OPTIS-An Einstein Mission for Improved Tests of Special and General Relativity. Gen. Relativ. Gravit. 2004, 36, 2373-2416.

4. Iorio, L.; Ciufolini, I.; Pavlis, E.C.; Schiller, S.; Dittus, H.; Lämmerzahl, C. On the possibility of measuring the Lense-Thirring effect with a LAGEOS LAGEOS II OPTIS mission. Classical. Quant. Grav. 2004, 21, 2139-2151.

5. Schiller, S.; Tino, G.M.; Gill, P.; Salomon, C.; Sterr, U.; Peik, E.; Nevsky, A.; Görlitz, A.; Svehla, D.; Ferrari, G. Einstein Gravity Explorer-a medium-class fundamental physics mission. Exp. Astron. 2009, 23, 573-610.

6. Turyshev, S.G.; Turyshev, S.G.; Sazhin, M.V.; Toth, V.T. General relativistic laser interferometric observables of the GRACE-Follow-On mission. Phys. Rev. D 2014, 89, 105029.

7. Everitt, C.W.F.; de Bra, D.B.; Parkinson, B.W.; Turneaure, J.P.; Conklin, J.W.; Heifetz, M.I.; Keiser, G.M.; Silbergleit, A.S.; Holmes, T.; Kolodziejczak, J.; et al. Gravity Probe B: Final Results of a Space Experiment to Test General Relativity. Phys. Rev. Lett. 2011, 106, 221101.

8. Ciufolini, I.; Pavlis, E.C. A confirmation of the general relativistic prediction of the Lense-Thirring effect. Nature 2004, 431, 958-960.

9. Ciufolini I.; Paolozzi A.; Pavlis, E.C.; Ries, J.C.; Koenig, R.; Matzner, R.A.; Sindoni, G.; Neumayer, H. Towards a One Percent Measurement of Frame Dragging by Spin with Satellite Laser Ranging to LAGEOS, LAGEOS 2 and LARES and GRACE Gravity Models. Space Sci. Rev. 2009, 148, 71-104.

10. Iorio, L.; Lichtenegger, H.I.M.; Ruggiero, M.L.; Corda, C. Phenomenology of the Lense-Thirring effect in the solar system. Astrophys. Space Sci. 2011, 331, 351-395.

11. Ciufolini, I. Frame Dragging and Lense-Thirring Effect. Gen. Relativ. Gravit. 2004, 36, 2257-2270.

12. Renzetti, G. History of the attempts to measure orbital frame-dragging with artificial satellites. Cent. Eur. J. Phys. 2013, 11, 531-544.

13. Soffel, M.; Wirrer, R.; Schastok, J.; Ruder, H.; Schneider, M. Relativistic effects in the motion of artificial satellites. I-The oblateness of the central body. Celest. Mech. 1988, 42, 81-89. 
14. Iorio, L. A possible new test of general relativity with Juno. Classical Quant. Grav. 2013, 30, 195011.

15. Iorio, L. Are we far from testing general relativity with the transitting extrasolar planet HD 209458b "Osiris" ? New Astron. 2006, 11, 490-494.

16. Iorio, L. Classical and relativistic long-term time variations of some observables for transiting exoplanets. Mon. Not. R. Astron. Soc. 2011, 411, 167-183.

17. Adams, F.C.; Laughlin, G. Relativistic Effects in Extrasolar Planetary Systems. Int. J. Mod. Phys. D 2006 15, 2133-2140.

18. Pal, A.; Kocsis, B. Periastron precession measurements in transiting extrasolar planetary systems at the level of general relativity. Mon. Not. R. Astron. Soc. 2008, 389, 191-198.

19. Jordan, A.; Bakos, G. Observability of the General Relativistic Precession of Periastra in Exoplanets. Astrophys. J. 2008, 685, 543-552.

20. Zhao, S.; Xie, Y. Parametrized post-Newtonian secular transit timing variations for exoplanets. Res. Astron. Astrophys. 2013, 13, 1231-1239.

21. Xie, Y.; Deng, X.-M. On the (im)possibility of testing new physics in exoplanets using transit timing variations: deviation from inverse-square law of gravity. Mon. Not. R. Astron. Soc. 2014, $438,1832-1838$.

22. Iorio, L. Accurate characterization of the stellar and orbital parameters of the exoplanetary system WASP-33b from orbital dynamics. Mon. Not. R. Astron. Soc. 2016, in press.

23. Iorio, L. Classical and relativistic node precessional effects in WASP-33b and perspectives for detecting them. Astrophys. Space Sci. 2011, 331, 485-496.

24. Goenner, H.F.M. On the History of Unified Field Theories. Living Rev. Relativ. 2004, 7 , 1830-1923.

25. Sciama, D.W. The Physical Structure of General Relativity. Rev. Mod. Phys. 1964, 36, 463-469.

26. Kibble, T.W.B. Lorentz invariance and the gravitational field. J. Math. Phys. 1961, 2, 212-221.

27. Mielke, E.W. Is Einstein-Cartan Theory Coupled to Light Fermions Asymptotically Safe? J. Grav. 2013, 2013, 5 .

28. Hehl, F.W. Gauge theory of gravity and spacetime. In Proceedings of the Workshop Towards a Theory of Spacetime Theories, Wuppertal, Germany, 21-23 July 2010.

29. Aldrovandi, R.; Pereira, J.G. Teleparallel Gravity; Springer: Berlin, Germany, 2013.

30. Hehl, F.W.; von der Heyde, P.; Kerlick, G.D.; Nester, J.M. General Relativity with Spin and Torsion: Foundations and Prospects. Rev. Mod. Phys. 1976, 48, 393-415.

31. Ni, W.T. Searches for the role of spin and polarization in gravity. Rep. Prog. Phys. 2010, 73, 056901.

32. Popławski, N.J. Cosmology with torsion: An alternative to cosmic inflation. Phys. Lett. B 2010, 694, 181-185.

33. Popławski, N.J. Non-singular, big-bounce cosmology from spinor-torsion coupling. Phys. Rev. D 2012, 85, 107502.

34. Obukhov, Y.N. Poincaré Gauge Gravity: Selected Topics; Cornell University: Ithaca, NY, USA, 2006.

35. Hammond, R.T. Torsion gravity. Rep. Prog. Phys. 2002, 65, 599-649. 
36. Hojman, S.; Rosenbaum, M.; Ryan, M.P.; Shepley, L. Gauge invariance, minimal coupling and torsion. Phys. Rev. D 1978, 17, 3141-3146.

37. Hojman, S.; Rosenbaum, M.; Ryan, M.P. Propagating torsion and gravitation. Phys. Rev. D 1979, $19,430-437$.

38. Hayashi, K.; Shirafuji, T. New General Relativity. Phys. Rev. D 1979, 19, 3524-3553.

39. Weitzenböck, R. Invariantentheorie; Popko Noordhoff: Groningen, The Netherlands, 1923.

40. Bel, L. Connecting Connections. A Bricklayer View of General Relativity; Cornell University: Ithaca, NY, USA, 2008.

41. Mao, Y.; Tegmark, M.; Guth, A.H.; Cabi, S. Constraining torsion with Gravity Probe B. Phys. Rev. D 2007, 76, 104029.

42. March, R.; Belletini, G.; Tauraso, R.; Dell'Agnello, S. Constraining spacetime torsion with the Moon and Mercury. Phys. Rev. D 2011, 83, 104008.

43. March, R.; Belletini, G.; Tauraso, R.; Dell'Agnello, S. Constraining spacetime torsion with LAGEOS. Gen. Relativ. Gravit. 2011, 43, 3099-3126.

44. Paolozzi, A.; Ciufolini, I. LARES successfully launched in orbit: Satellite and mission description. Acta Astronaut. 2013, 91, 313-321.

45. Iorio, L. The impact of the new Earth gravity models on the measurement of the Lense-Thirring effect with a new satellite. New Astron. 2005, 10, 616-635.

46. Renzetti, G. On Monte Carlo simulations of the LAser RElativity Satellite experiment. Acta Astronaut. 2015, 113, 164-168.

47. Ciufolini, I.; Moreno Monge, B.; Paolozzi, A.; Koenig, R.; Sindoni, G.; Michalak, G.; Pavlis, E.C. Monte Carlo simulations of the LARES space experiment to test General Relativity and fundamental physics. Class. Quantum Grav. 2013, 30, 235009.

48. Iorio, L. The impact of the orbital decay of the LAGEOS satellites on the frame-dragging tests, Adv. Space Res. 2016, in press.

49. Hehl, F.W.; Obukhov, Y.N. Élie Cartan torsion in geometry and in field theory, an essay. Annal. Found. Louis Broglie 2007, 32, 157-194.

50. Hehl, F.W.; Obukhov, Y.N.; Puetzfeld, D. On Poincaré gauge theory of gravity, its equations of motion, and Gravity Probe B. Phys. Lett. A 2013, 377, 1775-1781.

51. Kleinert, H.; Pelster, A. Autoparallels from a new action principle. Gen. Relativ. Grav. 1999, 31, 1439-1447.

52. Kleinert, H.; Shabanov, S.V. Spaces with Torsion from Embedding and the Special Role of Autoparallel Trajectories. Phys. Lett. B 1998, 428, 315-321.

53. Kleinert, H. Nonholonomic Mapping Principle for Classical and Quantum Mechanics in Spaces with Curvature and Torsion. Gen. Rel. Grav. 2000, 32, 769-839.

54. Anderson, J.D.; Nieto, M.M. Relativity in Fundamental Astronomy: Dynamics, Reference Frames, and Data Analysis; Klioner, S.A., Seidelmann, P.K., Soffel, M.H., Eds.; Proceedings IAU Symposium No. 261; Cambridge University Press: Cambridge, UK, 2010; pp. 189-197.

55. Lämmerzahl, C.; Preuss, O.; Dittus, H. Is the physics of the Solar System really understood? Lasers, Clocks Drag-Free Control 2008, 349, 75-101.

56. Iorio, L. Gravitational anomalies in the Solar System? Int. J. Mod. Phys. D 2015, 24, 1530015. 
57. Laskoš-Grabowski, P. The Einstein-Cartan Theory: The Meaning and Consequences of Torsion, Master's Thesis, University of Wrocław, Wrocław, Poland, 2009.

58. Puetzfeld, D.; Obukhov, Y.N. Probing non-Riemannian spacetime geometry. Phys. Lett. A 2008, $372,6711-6716$.

59. Landau, L.D.; Lifshitz, E.M. In The Classical Theory of Fields. Course of Theoretical Physics, 4th ed.; Butterworth-Heinemann: Oxford, UK, 1987; Volume 2.

60. Kleinert, H. Path Integrals in Quantum Mechanics, Statistics, Polymer Physics and Financial Markets, 15th ed.; World Scientific Publish. Co.: Singapore, Singapore, 2009.

61. Kleinert, H. Multivalued Fields: in Condensed Matter, Electromagnetism, and Gravitation; World Scientific Publ. Co.: Singapore, Singapore, 2008.

62. Kleinert, H.; Pelster, A. Novel Geometric Gauge Invariance of Autoparallels. Acta Phys. Pol. 1998, 29, 1015-1023.

63. Pollard, H. Mathematical Introduction to Celestial Mechanics; Prentice-Hall Inc.: Englewood Cliffs, NY, USA, 1966.

64. Danby, J.M.A. Fundamentals of Celestial Mechanics, 2nd ed.; Willmann-Bell, Inc.: Richmond, Virginia, USA, 1988.

65. Burns, J.A. Elementary derivation of the perturbation equations of celestial mechanics. Am. J. Phys. 1976, 44, 944-949.

66. Krasinsky, G.A.; Brumberg, V.A. Secular increase of astronomical unit from analysis of the major planet motions, and its interpretation. Celest. Mech. Dyn. Astron. 2004, 90, 267-288.

67. Standish, E.M. The astronomical unit now. In Transit of Venus: New Views of the Solar System and Galaxy; Kurtz, D.W., Ed.; Cambridge University Press: Cambridge, UK, 2005; p. 163.

68. Vinti, J.P. Classical solution of the two-body problem if the gravitational constant diminishes inversely with the age of the Universe. Mon. Not. R. Astron. Soc. 1974, 169, 417-427.

69. Iorio, L. An empirical explanation of the anomalous increases in the astronomical unit and the lunar eccentricity. Astron. J. 2011, 142, 68.

70. Acedo, L. Anomalous post-Newtonian terms and the secular increase of the astronomical unit. Adv. Space Res. 2013, 52, 1297-1303.

71. Li, X.; Chang, Z. Kinematics in Randers-Finsler geometry and secular increase of the astronomical unit. Chin. Phys. C 2011, 35, 914-919.

72. Miura, T.; Arakida, H.; Kasai, M.; Kuramata, S. Secular increase of the astronomical unit: A possible explanation in terms of the total angular momentum conservation law. Publ. Astron. Soc. Jpn. 2009, 61, 1247-1250.

73. Iorio, L. Secular increase of the astronomical unit and perihelion precessions as tests of the Dvali Gabadadze Porrati multi-dimensional braneworld scenario. J. Cosmol. Astropart. Phys. 2005, 2005,6 .

74. NASA Planetary Fact Sheet. Available online: http://nssdc.gsfc.nasa.gov/planetary/factsheet/ (accessed on 14 May 2015).

75. Acedo, L. Constraints on non-standard gravitomagnetism by the anomalous perihelion precession of the planets. Galaxies 2014, 2, 466-481. 
76. Iorio, L. A Critical Analysis of a Recent Test of the Lense-Thirring Effect with the LAGEOS Satellites. J. Geod. 2006, 80, 128-136.

77. Iorio, L.; Ruggiero, M.L.; Corda, C. Novel considerations about the error budget of the LAGEOS-based tests of frame-dragging with GRACE geopotential models. Acta Astronaut. 2013, 91, 141-148.

78. Renzetti G. Some reflections on the LAGEOS frame-dragging experiment in view of recent data analyses. New Astron. 2014, 29, 25-27.

79. Giles, P. Time-Distance Measurements of Large-Scale Flows in the Solar Convection Zone. Ph.D. Thesis, Stanford University, Stanford, CA, USA, 1999.

80. Stark, D.; Wöhl, H. On the solar rotation elements as determined from sunspot observations. Astron. Astrophys. 1981, 93, 241-244.

(c) 2015 by the author; licensee MDPI, Basel, Switzerland. This article is an open access article distributed under the terms and conditions of the Creative Commons Attribution license (http://creativecommons.org/licenses/by/4.0/). 